\title{
A!
}

This is an electronic reprint of the original article.

This reprint may differ from the original in pagination and typographic detail.

Badihi , Behnam; Zhao, Jianyu; Zhuang, Siyan; Seppänen, Olli; Jäntti, Riku

\section{Intelligent Construction Site: On Low Cost Automated Indoor Localization Using Bluetooth Low Energy Beacons}

Published in:

2019 IEEE Conference on Wireless Sensors, ICWiSe 2019

DOI:

10.1109/ICWISE47561.2019.8971829

Published: 01/11/2019

Document Version

Peer reviewed version

Please cite the original version:

Badihi , B., Zhao, J., Zhuang, S., Seppänen, O., \& Jäntti, R. (2019). Intelligent Construction Site: On Low Cost Automated Indoor Localization Using Bluetooth Low Energy Beacons. In 2019 IEEE Conference on Wireless Sensors, ICWiSe 2019 (pp. 29-35). [8971829] IEEE. https://doi.org/10.1109/ICWISE47561.2019.8971829

This material is protected by copyright and other intellectual property rights, and duplication or sale of all or part of any of the repository collections is not permitted, except that material may be duplicated by you for your research use or educational purposes in electronic or print form. You must obtain permission for any other use. Electronic or print copies may not be offered, whether for sale or otherwise to anyone who is not an authorised user. 


\title{
Intelligent Construction Site: On Low Cost Automated Indoor Localization Using Bluetooth Low Energy Beacons
}

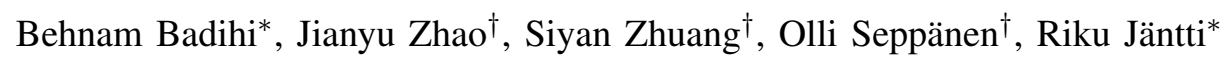 \\ * Department of Communication and Networking \\ ${ }^{\dagger}$ Department of Civil Engineering \\ Aalto University, Espoo, 02150 Finland \\ Email:\{firstname.lastname\}@aalto.fi
}

\begin{abstract}
The construction jobsites are highly dynamic environments involved with labor-intensive and manual tasks that suffer from inefficiency and mis-management. To remedy these issues, production control within the scope of lean construction has been a hot topic in academia and industry. To this end, we have developed a real-time coordination-based tracking system using low-cost Bluetooth Low Energy beacons. This system is implemented according to the trilateration method in order to acquire the real-time location of resources and movement trajectory in the construction jobsites. The prototype is tested and implemented in the real construction site in China. The results show that the implemented tracking system can be used in the construction sites depending on the accuracy level required in the project.
\end{abstract}

Index Terms-intelligent construction site, indoor positioning, Bluetooth Low Energy, Onsite management, automated tracking system

\section{INTRODUCTION}

$\mathbf{T}$ he construction site is a complicated and dynamic environment characterized by various interactions between labor, material, and tools. As a major contributor to the world economy, the construction industry is plagued by poor productivity and high level of inefficiency resulting from resource mis-allocation. The mismanagement, in turn, causes over-using or under-using the resources in the construction sites [1], [2]. Many studies confirm the reduction of efficiency in the construction sites in the last few decades [3], [4]. Besides, this industry is very labor-intensive involving manual, repetitive and physical tasks with exposure to high degree of risk and health issues [5].

To assuage these issues, it is necessary to obtain real-time information from the labors, materials, and equipment. The location data can be further processed to evaluate the quality of workflow and eventually to disclose the efficiency of the work. All in all, real-time location information of material, labor and equipment are valuable for measuring the efficiency, consistent workflow and safety.

Even though a wide range of technologies have been utilized for position and tracking, yet the analysis of productivity has been manually conducted [6]. Furthermore, there is a lack of affordable, easy-to-use, and easy-to-deploy solution that fulfills the gap between online tracking information and management concerns, in particular, in terms of jobsite safety, productivity control, and supply chain. To this end, we have developed a low cost, scalable, and easy-to-deploy solution for automated tracking by using Bluetooth Low Energy (BLE) Beacons. We have deployed and tested this solution in our test-bed and construction sites in China.

\section{Previous Studies}

To enhance the onsite operation efficiency and eliminate waste, lean construction methods have been discussed and applied in many construction projects [7]. Those approaches such as the Location-Based Management System (LBMS) [8], Takt time planning [9] and Last Planner System (LPS) [10] have not been developed with automated data collection technology in mind, and they have not been able to solve the substantial time and labor efforts invested for an effective operations management in construction industry [11]. Therefore, the next generation of production control framework in construction should integrate a system where automated data input could play an important role in improving the onsite management and monitoring process [12].

The tracking solutions [11] have served different purposes of onsite management requirement but they all nevertheless aim at promoting the transition from traditional onsite management towards more intelligent and automated platforms [13]. Among many indoor tracking technologies, some of them have been already successfully implemented in real construction projects such as ZigBee [14], RFID [15], Ultra-wideband (UWB) [2], and Bluetooth Low Energy (BLE) [16]. BLE has successfully been implemented in several real construction projects in order to measure and analyze the presence level of workers onsite correlated with the value-adding time of workers [11]. However, the presented tracking system could only determine the location information of the closest gateway to which the beacons broadcast their signals. That could be problematic when the lack of location details might reflect inaccurately upon the value-adding activities onsite. Therefore, there is a need for improving the tracking systemso that the actual value-adding movements of workers onsite can be accurately identified.

Trilateration with BLE beacons for indoor positioning has been a popular research topic that has also been applied to the 


\section{BLE Beacons}

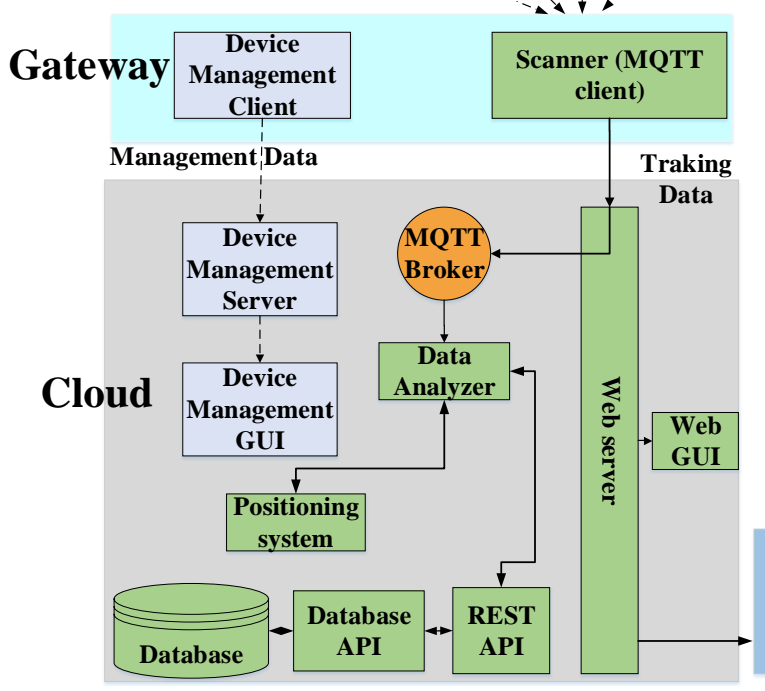

Despite the known challenges with signal fluctuation, trilateration with RSSI was the natural next step to increase the precision of our existing BLE positioning system [16], because it did not require extra hardware equipment and could be achieved merely by implementing the algorithm on top of the system. In this paper, we implemented the trilateration method in our already existing location tracking platform (based on the Cell of Origin method) to increase the accuracy of the tracking system. This system has been tested in the real construction site with a harsh indoor environment.

\section{StRucture OF The SOlution}

\section{A. Architecture}

We introduce the architecture of our tracking and positioning system in this section. This system comprises of three separate areas: a) Bluetooth Low Energy (BLE) beacons, b) gateways, and c) cloud. In addition, there are two independent data-flow in this solution as tracking data plane and management plane. The overview of our tracking and positioning system is presented in Fig. 1. The details of the solution are provided in the following.

1) BLE Beacons: BLE beacon uses Bluetooth Low Energy (BLE) radio technology [24] to send data to the surrounding environment. This can be compared to a lighthouse that emits light and therefore can be observed in the vicinity. Beacons similar to the concept of lighthouse broadcast the data to their surroundings. The signal can be captured by any compatible device. We have used a dedicated beacon for each individual in our solution. Since each beacon has a unique MAC (media access control) address, we have utilized the MAC address and mapped each individual to that specific MAC address. By mapping the MAC address and individual in the database, the individuals can be identified in the cloud. Figure 2 shows the beacons that are used in our tracking system.

2) Gateway: The broadcasted signals of the beacons are collected and aggregated by the gateways. The aggregated data is transmitted to the cloud in an adjustable time interval. In other words, the gateways play the role of intermediate entities between beacons and the cloud. The connectivity of the gateways is provided by WiFi or cellular technology.

3) Cloud: The heart of our tracking and positioning solution lies in the cloud. The gateways send their tracking data to the cloud using Message Queuing Telemetry Transport (MQTT) protocol [25]. MQTT is a publish- subscribe based messaging protocol in which clients communicate with the server which is often called broker. The clients located in the gateways publish the data and send it to the broker located in the cloud. Data Analyzer, one of the modules in the cloud, subscribes to the broker and consumes the data directly coming from the gateways. Data Analyzer is the brain of the cloud, responsible for analyzing incoming data, filtering out redundant information, selecting the gateway for the beacons, and storing data in the database. Furthermore, Data Analyzer provides the required data for the Positioning module. The positioning module determines the coordination of the beacons relative to one of the gateways in the system as a reference point. 
The major role of the Data Analyzer is to select the closest (distance-wise) gateway to a given beacon used at the site. This is done by using the RSSI of the beacons. The gateways measure and collect the RSSI of the beacons and send them to the cloud. Upon the receiving of these values, Data Analyzer analyzes and compares the RSSI values of the beacons reported by several gateways and selects the gateway for the given beacons based on the highest RSSI. The locations of the gateways are known at the site. By knowing the selected gateway for a given beacon, the location of the beacon is determined. This method of positioning is known as the Cell of Origin [26]. In this method, each gateway assumed as a cell has a predefined coverage. This coverage is determined by the maximum transmission range of the beacons, which is assumed to be the same for all beacons. Once a given beacon is mapped to a given gateway, since the location of the gateway is known therefore the location of the beacon is known.

Once the locations of the beacons are determined, the Data Analyzer stores this information in the database using the Database API (Application Programming Interface) module.

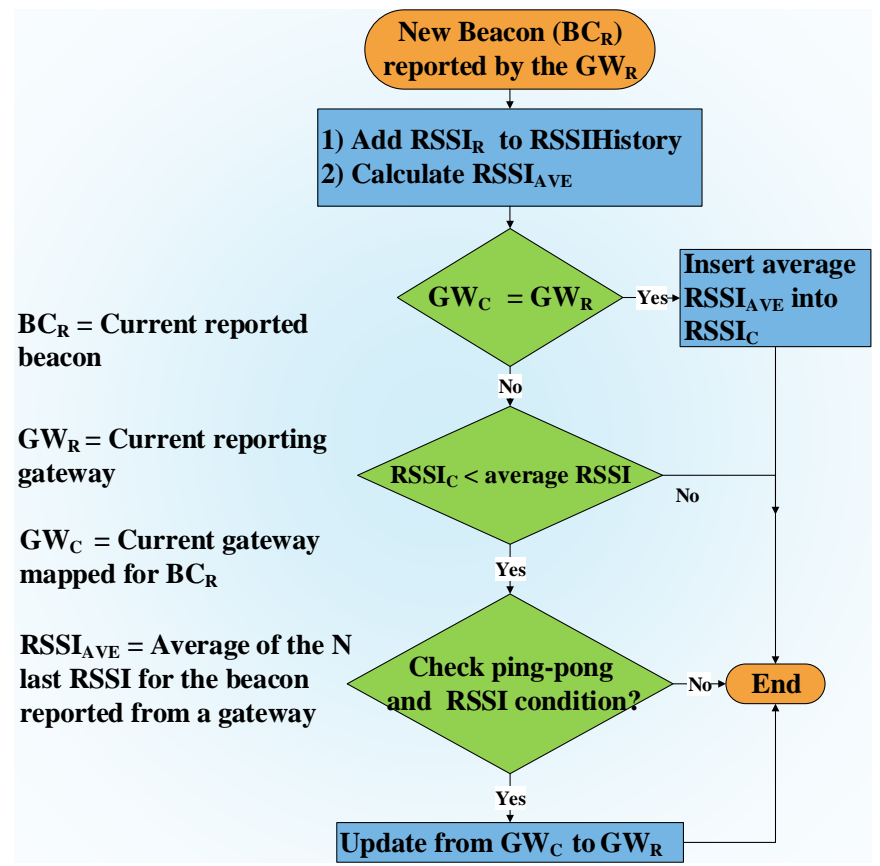

Fig. 3. Data Analyzer algorithm.
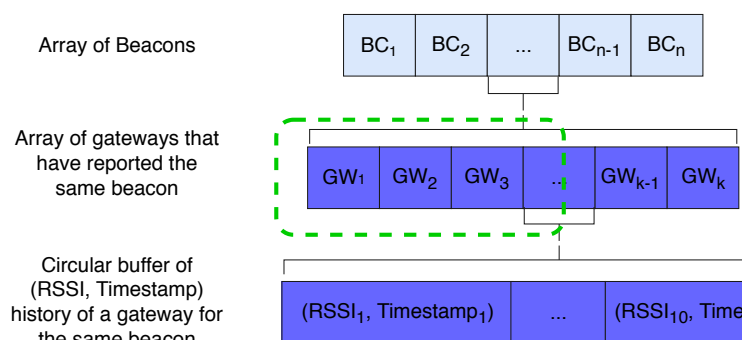

tory of a gateway for

the same beacon

Fig. 4. Data types modelling the beacon-to-gateway connections. The green dotted box shows the three nearest gateways detecting a beacon, which are used for trilateration.

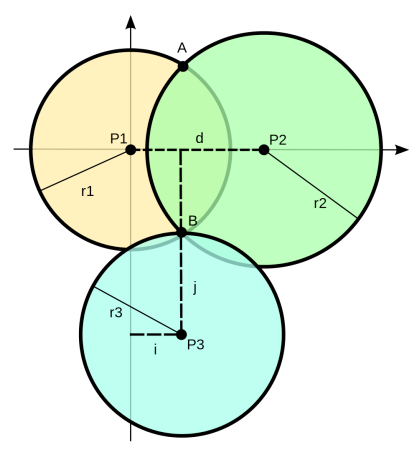

Fig. 5. Trilateration in xy-plane; $d_{1}, d_{2}$, and $d_{3}$ can be calculated from the respective RSSI values.

The stored data is consumed by third party applications using REST (Representational State Transfer) API and visualized using WEB or mobile applications.

\section{B. Tracking and positioning algorithm}

Data Analyzer adopts the algorithm depicted in Fig. 3 in order to implement the Cell of Origin method. This algorithm records the history of the RSSI of the beacons reported from the gateways. In other words, there is more that one buffer of RSSI history for a single beacon reported by the gateways which can hear that beacon. In this way, several parallel histories of RSSI, which are reported by several gateways, can be recorded for a single beacon. These RSSI values are associated with their timestamps. These timestamps are used to remove the RSSIs that arrived before a predetermined time duration. In this way, only those RSSIs are kept which reported within a predetermined time duration. This is vital for comparing the average RSSI of one gateway to the average RSSI of another gateway for a given beacon. The data structure for recording RSSI history is depicted in Fig. 4. Furthermore, in order to reduce the ping-pong effect, the cell (gateway) is only changed for a beacon when the average RSSI for the reporting gateway is higher than the RSSI reported by the currently associated gateway plus a margin, which is also called hysteresis margin. Ping ping effect occurs when the beacons are at the edge of two or more cells (gateways) and the associated cell (gateway) for the beacon is repeatedly changed between the cells [27].

On top of the beacon positioning based on the (Cell of Origin method), a coordinate-level location tracking is implemented according to the trilateration method. Trilateration requires the knowledge of at least three gateways detecting a single beacon (see Fig. 5). The coordinates of the gateways are known, so the distances of a beacon to three distinct fixed points can be determined. The origin of the coordinate system can be arbitrarily defined, for example as a corner of the building.

The theoretical relationship between RSSI and the signal detection distance $d$ can be calculated by an indoor radio signal propagation model that takes into account the reference transmission power $A$ and the environmental factor $n$ [19].

$$
R S S I=A-10 n \log d
$$




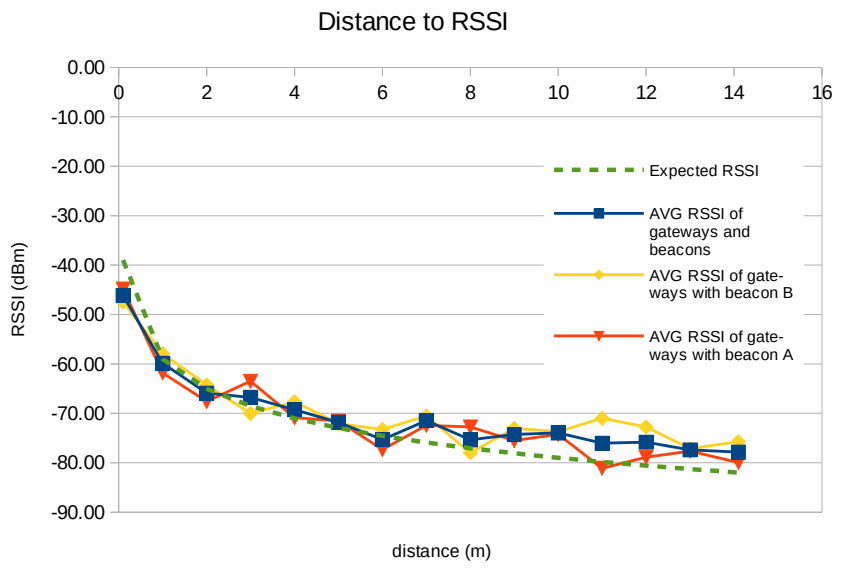

Fig. 6. Distance to RSSI relationship. Dotted green line shows the expected curve when $n=2$.

The reference transmission power $A$ represents the RSSI at distance $d=1$ meter [23]. The environmental factor $n$ indicates the attenuation of the radio signal due to the environment, and thus it is also referred to as the attenuation factor [23] and usually determined experimentally [19] [28].

For this system, $n=2$ was selected after experiments determining the distance-to-RSSI relationship. Fig. 6 shows the distance-to-RSSI measurements with two co-located gateways and two beacons. The yellow and the red data sets show the measurements for individual beacons, while the blue data set shows their average. The transmission power was set such that $A=-59 \mathrm{dBm}$.

Selecting the nearest three gateways for a beacon is based on the average RSSI value of the gateways detecting this beacon. This average RSSI value for a gateway is calculated from the latest three RSSI values in the history buffer of this beacon-to-gateway connection (see Fig. 4, circular buffer). Thus trilateration takes three nearest gateways as the input

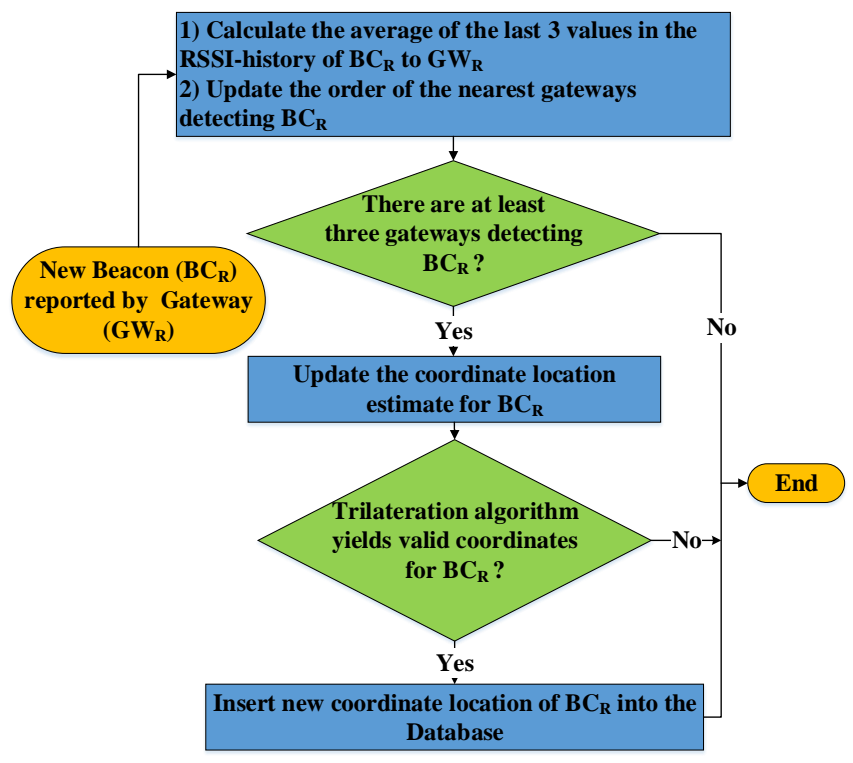

Fig. 7. Positioning algorithm determined by the average RSSI values.

The trilateration algorithms are implemented in the Positioning System module (refer to Fig. 7). Two trilateration algorithms are implemented and tested. The first one is the Least Square Estimation (LSE) and the other one is the Centroid algorithm [19]. LSE turned out to be more accurate when it yields coordinate values, but the Centroid algorithm yields coordinate values more often than LSE. LSE suffered from a low output rate, as it was less tolerant for measuring errors. Centroid algorithm was more resilient. With the general degree of inaccuracy of the system, the slightly more accurate output of LSE was unimportant. The Centroid algorithm was more reliable in total and thus chosen.

The Centroid algorithm is based on finding the centroid of the polygon confined by the points of inner intersections of the circles with radius indicating the measured distance from the beacon to each of the three gateways. There are three ways of how three circles may intersect with each other (see Fig. 8). When there are no intersections, the algorithm does not yield any coordinate.

\section{RESULTS AND DISCUSSION}

\section{A. Case study}

The location tracking system was tested in a construction site in Shijiazhuang, China. The case was a large shopping mall that was under the mechanical, electrical and plumbing (MEP) installation phase. The second floor is a large open space without many load-bearing walls, but rather with a regular grid of load-bearing pillars (see Fig. 9). This was a suitable real construction site environment for testing the trilateration-based tracking system because of the absence of walls within a floor. Bose et al. (2007) shows that when there is not line-of-sight between a transmitter and a receiver, the multipath effect increases, which makes distance estimation from RSSI more difficult [28]. This study was meant to test the trilateration system first in the simplest possible (but yet common) construction site layout.

Seven gateways were attached to the building pillars onsite. Each adjacent pillar is nine meters away from the other. We set point D2C2 from the blueprint (not shown in the pictures) as the origin $(0,0)$, and all gateways received their coordinates with respect to it (see Fig. 10 to 12). To be able to test the system, we have conducted several experiments on the site:

1) Decide one fixed path connected with 20 test points for one to walk within the area where the gateways are installed.

2) Walk following the path with different time intervals that are used to stay at each test point. The chosen time intervals are 30 seconds, 1 minute and 2 minutes at each test point. Therefore one round of walking took 10 minutes, 20 minutes and 40 minutes, respectively (see Fig. 10 to 12 ).

3) Given the system configuration parameters where the coordinates of the beacons were sent in second time interval, the coordinate data of beacons need to be averaged to fit in our chosen test points. We first filter out obvious error coordinates (X-axis larger than 100 or smaller than 0 , or 


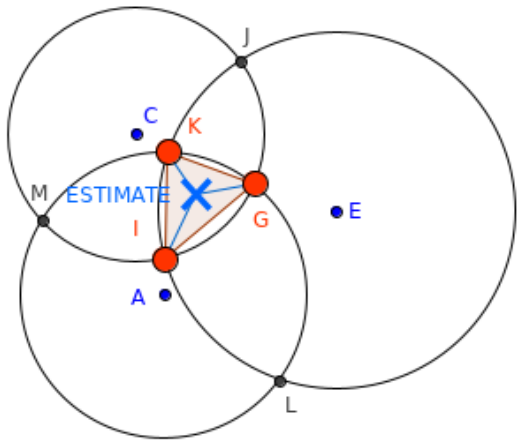

(a)

Fig. 8. Centroid algorithm scenarios.

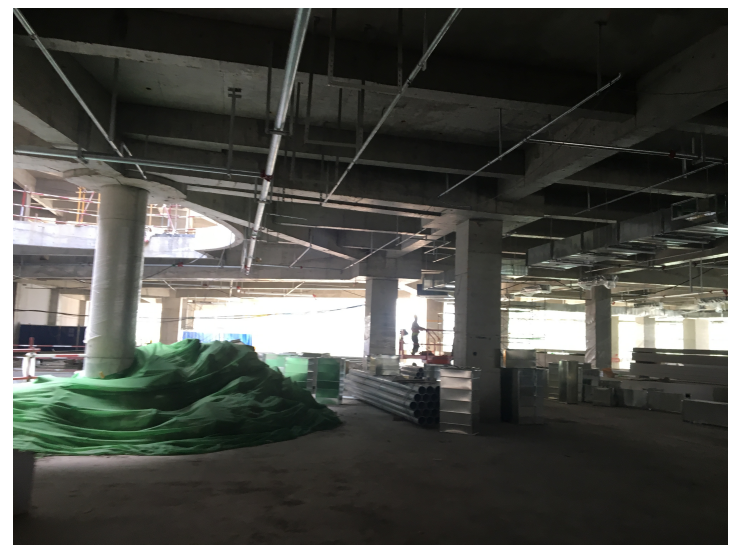

Fig. 9. Photo from Shijiazhuang construction site

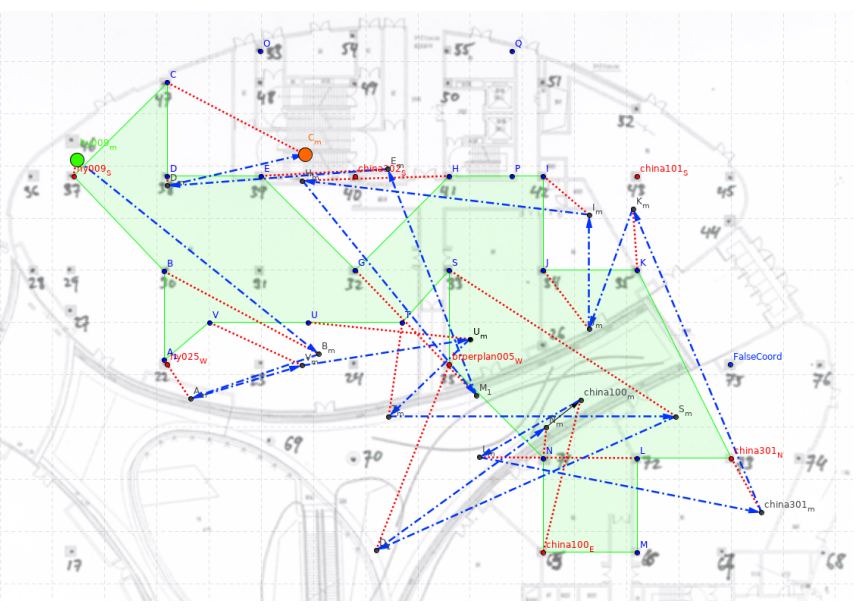

Fig. 10. Validation with 2 min intervals, average points

Y-axis larger than 100 or smaller than 0 ); then we filter out the first 5 seconds and last 10 seconds of each time intervals because it is likely at that time the beacon was moving from one place to another; Finally we average the coordinates in each test time intervals and determine the averaged system-recorded coordinates of each chosen

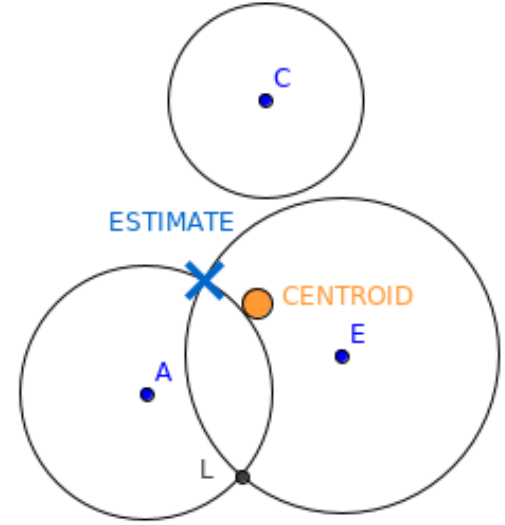

(c) (b)

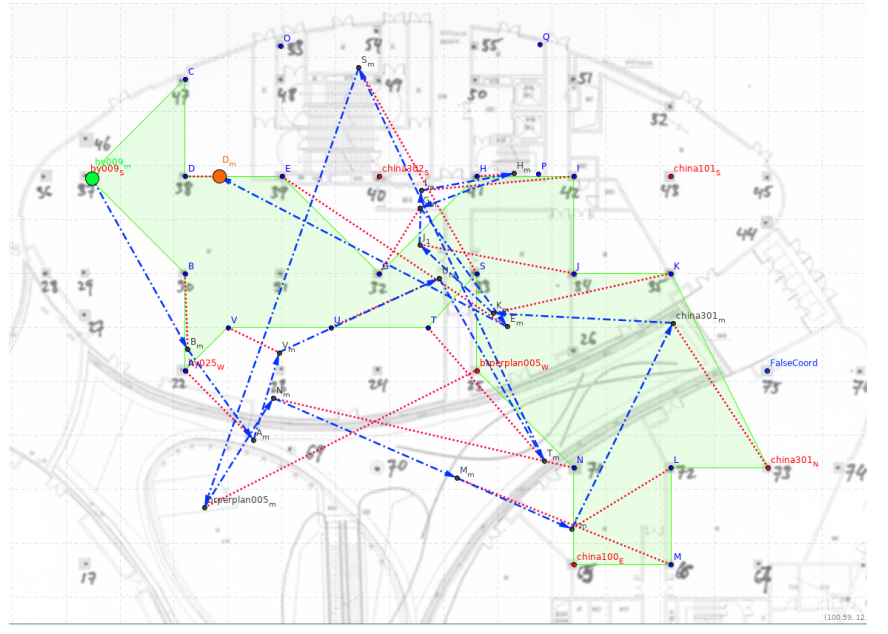

Fig. 11. Validation with 1 min intervals, average points

test point.

4) Reduce the movement line in the system based on the averaged system-recorded coordinates of the chosen test point and examine how accurate the tracking system could capture the movement on site.

Fig. 10 to 12 show the results of three validation rounds. The edges of the green polygon show the validation path, and the blue and red points along it are the measuring points. The path and the measuring points were the same for each validation round. The person walking along the validation path stopped at each measuring point for 2 minutes in the first round, 1 minute in the second round, and 30 seconds in the third round. In these three figures, for each measuring point, the averages of the recorded points (each recorded point calculated by trilateration) are taken and marked in the figure as a single point in the dark. The blue decorated line shows the output path formed by connecting the average points, and the red densely dotted lines represent the differences of the average points to the real measuring points. Fig. 13 shows the last validation round ( $30 \mathrm{sec})$, but unlike Fig. 12, it shows all nonrepetitive recorded points instead of the average measurements 


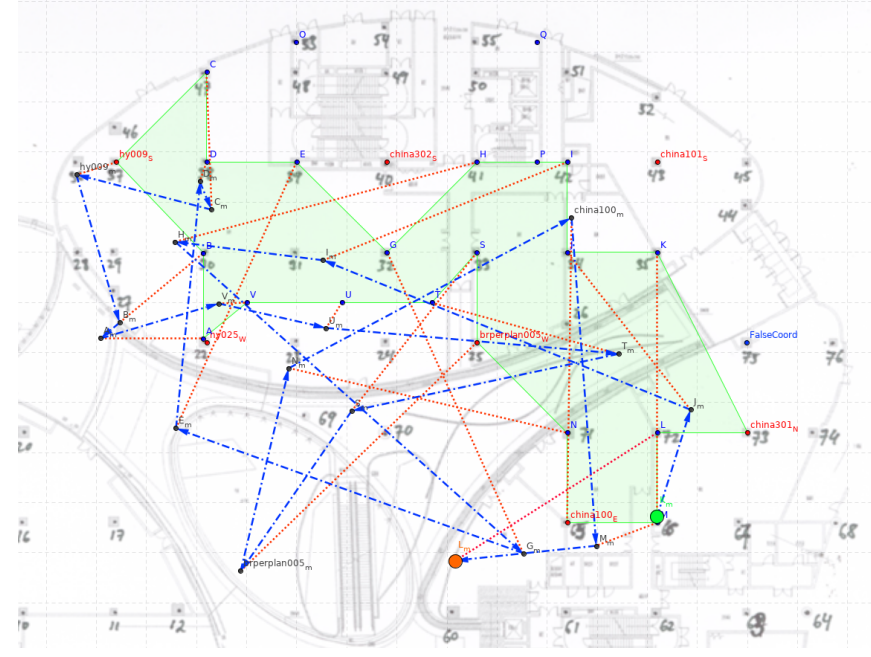

Fig. 12. Validation with $30 \mathrm{sec}$ intervals, average points

\begin{tabular}{lllll}
\hline Validation round & $\begin{array}{l}\text { X-coordinate } \\
\text { correlation }\end{array}$ & $\begin{array}{l}\text { y-coordinate } \\
\text { correlation }\end{array}$ & $\begin{array}{l}\text { AVG deviation } \\
\text { of all recorded } \\
\text { points }(\mathrm{m})\end{array}$ & $\begin{array}{l}\text { Standard deviation } \\
\text { of all recorded } \\
\text { points }(\mathrm{m})\end{array}$ \\
\hline $2 \mathrm{~min}$ & 0.86 & 0.81 & 10.69 & 6.72 \\
$1 \mathrm{~min}$ & 0.67 & 0.71 & 14.06 & 9.71 \\
$30 \mathrm{sec}$ & 0.74 & 0.35 & 19.18 & 11.62 \\
\hline
\end{tabular}

TABLE I

RESULTS OF THE VALIDATIONS ROUNDS WITH 2 MIN, 1 MIN, AND 30 SEC MEASUREMENT INTERVALS

at each measuring point.

As the figures show, the 2 min validation round achieved the highest accuracy, while the 1 min validation round yielded the least accurate results. The deviation of each validation round are presented in Table I.

\section{B. Potential use cases in construction}

One important managerial implication regarding the coordinate-integrated tracking system applied in indoor construction projects is to obtain more accurate information on value-adding activities automatically onsite from the workers.

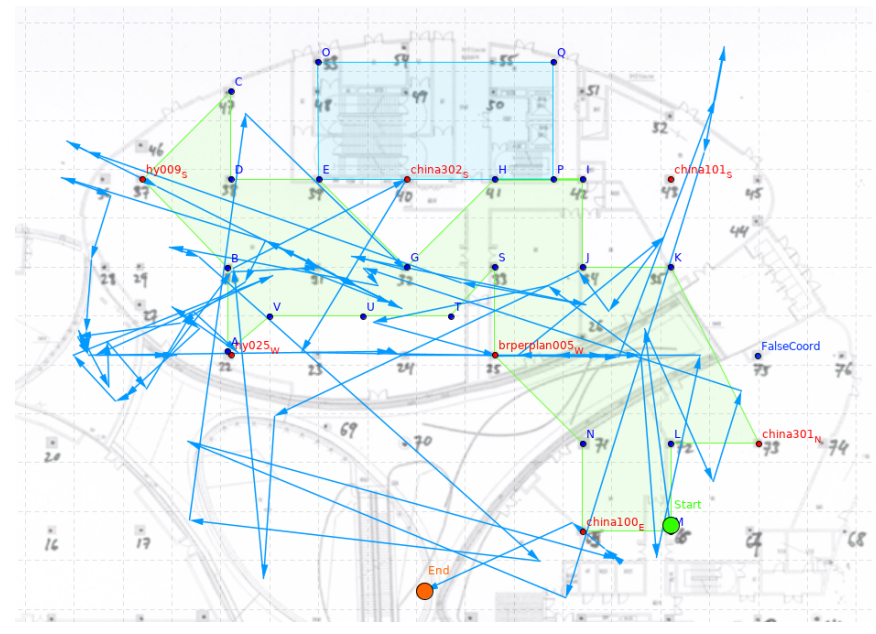

Fig. 13. Validation with $30 \mathrm{sec}$ intervals, unique points
Zhao et. al [11] have argued that the presence level of workers onsite should be correlated to their value-adding level and the BLE-based location tracking system they applied is one way to automate the data collection process. However, in their paper, the installed gateways could only detect and identify which beacons are closest using the cell of origin method. The current coordinates of workers onsite with time stamps enable tracking the task flow lines thus leading to more accurate and automated value-adding task movement detection. For instance, in this case where we tracked the MEP workers doing the pipeline installation, the coordinates of workers on the site could be visualized for checking if the system recorded movement matches the real pipeline installation direction (Fig 9), and how deviant the movement line could make influence on the daily productivity of the pipe installation. Furthermore, coordinates can be used for generating heat-maps of working areas onsite, which can outline the actual working edges with different colors indicating onsite presence levels.

The coordinate feature of the system can also enhance the tool management onsite for workers. Workers often spend much wasteful time looking for the right tools. With the help of more accurate positioning information of their tools, the workers could identify them faster in the chaotic environment. Furthermore, tools could move only when they are in use and this helps site managers more precisely estimate the valueadding level of the workers performing the tasks.

\section{Discussion and future work}

Given our attempts to improve the BLE-based tracking system applied in construction with coordinate features using trilateration technology, we aim to answer the following research questions in the paper: How does trilateration improve the existing BLE Beacon based location tracking system? How can a real-time tracking system with coordinate positioning feature help operation management in construction? And what are the use cases in construction for this prototype?

Our trilateration based coordinate-level location tracking reaches the accuracy of at best 10.69 meters in a real construction environment ( 2 min case). Park et al. (2016) achieved an excellent accuracy of less than 2 meters error with probabilitybased algorithms with BLE combined with motion sensors. However, the beacon density was not specified [18]. Wang et al. (2013) conducted the positioning experiments in a $6 \mathrm{~m}$ x $8 \mathrm{~m}$ room with four beacons at the corners and reached an accuracy of less than $40 \mathrm{~cm}$ of error. Here mobile phone was used as the tracked object, whereas the beacons here had the same role as the gateways in our system [19]. Bose et al. (2007) reached the accuracy of less than $3 \mathrm{~m}$ of error with WiFi in an ordinary indoor environment with walls [28]. Compared to these results, our results seem very inaccurate. However, the technology and the environments in these studies were more or less different from ours. Out of these three references, Park et al. (2016) is the most comparable to our study as it shares the same type of environment and technology, although their additional use of motion sensors makes a large difference.

The current coordinate-level location tracking can be used in the projects with different levels of the requirements on 
the accuracy. The accuracy of the current system depends on the movement trajectory as well. Also, the density of the deployed gateways affects accuracy. In this study, we placed gateways 15 to 20 meters apart, because of a limited amount of gateways in use. By deploying gateways more densely, more accurate results will be expected. The requirement for accuracy of the coordination is subject to the degree of the waste to be controlled in the project. As an example, this system can be beneficiary for tracking big tools and machinery in the construction sites when the error in meter scale can be negligible.

The future work regarding the localization is to even further increase the accuracy of the coordination system by using the new technology and techniques such as using Angle of arrival (AoA) and Angle of departure (AoD) methods. These methods are standardized in BLE version 5.1 and soon will be utilized in the devices.

\section{Conclusion}

The construction industry as a major contributor to the world economy is currently undergone massive efficiency and waste due to managerial reasons and resource misallocations. To rectify these issues, obtaining real time information from the laborers, materials, and equipment is essential. In this regard, we have developed and implemented a real-time coordinate-level location tracking system utilizing the low-cost BLE beacons. The localization method in this work is based on the trilateration method. The corresponding algorithms are developed to use the received signal strength from the beacons and calculate the coordination of the trajectories in the construction environments. Three rounds of validation experiments are held in the real construction site in China. The study shows that the current setup can be beneficiary in the construction sites to obtain the real-time location of the workers and materials to obtain managerial information regarding the waste in the construction sites.

\section{REFERENCES}

[1] R. Akhavian and A. H. Behzadan, "Smartphone-based construction workers' activity recognition and classification," Automation in Construction, vol. 71, pp. 198-209, 2016.

[2] T. Cheng, J. Teizer, G. C. Migliaccio, and U. C. Gatti, "Automated tasklevel activity analysis through fusion of real time location sensors and worker's thoracic posture data," Automation in Construction, vol. 29, pp. 24-39, 2013

[3] P. Teicholz, "Labor-productivity declines in the construction industry: causes and remedies (a second look)," AECbytes Viewpoint, 2013.

[4] D. Arditi and K. Mochtar, "Trends in productivity improvement in the us construction industry," Construction Management \& Economics, vol. 18 no. 1, pp. 15-27, 2000.

[5] V. Arndt, D. Rothenbacher, U. Daniel, B. Zschenderlein, S. Schuberth, and $\mathrm{H}$. Brenner, "Construction work and risk of occupational disability: a ten year follow up of 14474 male workers," Occupational and environmental medicine, vol. 62, no. 8, pp. 559-566, 2005.

[6] S. Taneja, B. Akinci, J. H. Garrett, L. Soibelman, E. Ergen, A. Pradhan, P. Tang, M. Berges, G. Atasoy, X. Liu et al., "Sensing and field data capture for construction and facility operations," Journal of construction engineering and management, vol. 137, no. 10, pp. 870-881, 2010.

[7] O. Seppänen, R. Modrich, and G. Ballard, "Integration of last planner system and location-based management system," in In: Proc. 23 rd Ann. Conf. of the Int'l. Group for Lean Construction, 2015, pp. 123-132.

[8] R. Kenley and O. Seppänen, "Location-based management for construction: Planning," 2010.
[9] A. Frandson, K. Berghede, and I. D. Tommelein, "Takt time planning for construction of exterior cladding," in Proc. 21st Ann. Conf. of the Intl Group for Lean Construction, 2013.

[10] C. T. Formoso and C. B. Moura, "Evaluation of the impact of the last planner system on the performance of construction projects," in Proceedings of 17th Annual Conference of the International Group of Lean Construction, 2009, pp. 153-164.

[11] J. Zhao, O. Seppänen, A. Peltokorpi, B. Badihi, and H. Olivieri, "Realtime resource tracking for analyzing value-adding time in construction," Automation in Construction, vol. 104, pp. 52-65, 2019.

[12] T. Nath, M. Attarzadeh, R. L. Tiong, C. Chidambaram, and Z. Yu, "Productivity improvement of precast shop drawings generation through bim-based process re-engineering," Automation in Construction, vol. 54, pp. 54-68, 2015 .

[13] H. Olivieri, O. Seppänen, A. Peltokorpi et al., "Real-time tracking of production control: requirements and solutions," in In: Proc. Lean \& Computing in Construction Congress (LC3), vol. 1, 2017.

[14] P. Lin, Q. Li, Q. Fan, and X. Gao, "Real-time monitoring system for workers' behaviour analysis on a large-dam construction site," International Journal of Distributed Sensor Networks, vol. 9, no. 10, p. 509423, 2013

[15] A. Costin, N. Pradhananga, and J. Teizer, "Leveraging passive rfid technology for construction resource field mobility and status monitoring in a high-rise renovation project," Automation in Construction, vol. 24, pp. 1-15, 2012.

[16] J. Zhao, H. Olivieri, O. Seppänen, A. Peltokorpi, B. Badihi, and P. Lundström, "Data analysis on applying real time tracking in production control of construction," in 2017 IEEE International Conference on Industrial Engineering and Engineering Management (IEEM). IEEE, 2017, pp. 573-577.

[17] J. Park, K. Kim, and Y. K. Cho, "Framework of automated constructionsafety monitoring using cloud-enabled bim and ble mobile tracking sensors," Journal of Construction Engineering and Management, vol. 143, no. 2, p. 05016019, 2016

[18] J. Park, Y. K. Cho, and C. R. Ahn, "A wireless tracking system integrated with bim for indoor construction applications," in Construction Research Congress 2016, 2016, pp. 2660-2668.

[19] Y. Wang, X. Yang, Y. Zhao, Y. Liu, and L. Cuthbert, "Bluetooth positioning using RSSI and triangulation methods," 2013 IEEE 10th Consumer Communications and Networking Conference, CCNC 2013, pp. 837-842, 2013.

[20] J. Park, J. Kim, and S. Kang, "BLE-Based Accurate Indoor Location Tracking for Home and Office," Computer Science \& Information Technology (CS \& IT), pp. 173-181, 2015.

[21] T. Cheng, M. Venugopal, J. Teizer, and P. A. Vela, "Performance evaluation of ultra wideband technology for construction resource location tracking in harsh environments," Automation in Construction, vol. 20, no. 8, pp. 1173-1184, 2011. [Online]. Available: http://dx.doi.org/10.1016/j.autcon.2011.05.001

[22] V. C. Paterna, A. C. Augé, J. P. Aspas, and M. A. P. Bullones, "A bluetooth low energy indoor positioning system with channel diversity, weighted trilateration and kalman filtering," Sensors (Switzerland), vol. 17 , no. $12,2017$.

[23] Z. Jianyong, L. Haiyong, C. Zili, and L. Zhaohui, "Rssi based bluetooth low energy indoor positioning," in 2014 International Conference on Indoor Positioning and Indoor Navigation (IPIN). IEEE, 2014, pp. 526-533.

[24] Bluetooth Core Specification, The Bluetooth Special Interest Group, https://www.bluetooth.com/ specifications/bluetooth-core-specification.

[25] MQTT Version 5.0 specification, 07 march 2019. [Online]. Available: https://docs.oasis-open.org/mqtt/mqtt/v5.0/mqtt-v5.0.html

[26] H. Li and H. Ma, "A low complexity low power indoor positioning system based on wireless received signal strength," in 2018 IEEE 20th International Conference on e-Health Networking, Applications and Services (Healthcom), Sep. 2018, pp. 1-6.

[27] R. P. Ray and L. Tang, "Hysteresis Margin and Load Balancing for Handover in Heterogeneous Network,' International Journal of Future Computer and Communication, vol. 4, no. 4, pp. 231-235, 2015.

[28] A. Bose and H. F. Chuan, "A practical path loss model for indoor WiFi positioning enhancement," 2007 6th International Conference on Information, Communications and Signal Processing, ICICS, pp. 0-4, 2007. 